\title{
Multi-factors Optimization Design of Pin-Fin Structure Using Respond Surface Method
}

\author{
Xiang Wang ${ }^{\mathrm{a}}$, Min Chen ${ }^{\mathrm{b},}$, , Derrick Tate ${ }^{\mathrm{c}}$ \\ Department of Industrial Design, Xi’an Jiaotong-Liverpool University, Suzhou, China \\ a xiang. wang@student.xjtlu.edu.cn , b min.chen@xjtlu.edu.cn, ${ }^{\mathrm{c}}$ d.tate@xjtlu.edu.cn \\ *Corresponding author
}

Keywords: Response surface method, pin-fin, optimization, pressure loss, thermal resistance

\begin{abstract}
This study aims to find out the optimized solution for coupled effects of parameters. Different turbulence models were studied and RSM (Response Surface Methodology) was the most appropriate one to deal with optimization of target and investigate interaction of different parameters. In design height and diameter of cylindrical pin-fin was adopted as control parameters, according to numerical simulation the thermal resistance and pressure loss of pin-fin heat sink indicated improving tendency with increasing the both parameters. Sensitivity analysis was conducted to investigate the correlation of two geometrical parameters to response target. Less head loss and better cooling efficiency were achieved from an optimized structure.
\end{abstract}

\section{Introduction}

Pin-fin heat sink in different shape is widely applied in various electronic devices that generate a large amount of heat, such as power control unit (PCU) of vehicles and central processing unit (CPU), to extend the lifespan and reliability of devices by improving efficiency of heat transfer. The requirement for traditional electronic equipment generally can be fulfilled by basic design of fins, such as adopting plate fins and square fins without modification. Nevertheless, development of electronic technology results in the increase of power dissipation and makes more rigorous demand for performance of pin-fin heat sink, therefore multitudinous optimized methods aiming at the performance of fins are proposed and investigated ${ }^{[1]}$.

There are several factors, such as materials, geometry or distribution of pin-fins, affecting the heat dissipation and hydraulic performance of the cooling system. Ricci et al. ${ }^{[2]}$ conduct the experiment to explore the hydraulic features of the heat sink with various shapes of pin-fin (cylindrical, square and triangular prism) distributed inline. The influence of inline and staggered arrangement on thermal performance of pin-fin heat sink was investigated through experiment, and optimization scheme was achieved $^{[3]}$. Similarly, Rubio-Jimenez et al ${ }^{[4]}$ numerically investigate the influence of pin-fin density with both online and staggered distribution. Zhao ${ }^{[5]}$ investigated the effects of square pin-fin density and angle on thermal performance, and obtain the optimal design. Furthermore, the geometrical characteristic also affects the performance of pin-fin heat sink. Cormier ${ }^{[6]}$ demonstrated the increase of fins height and density have positive influence on the thermal conductance and negative effects on hydraulic performance of heat sink. Joshi et al. ${ }^{[8,7]}$ experimentally study the influence of major pin-fin parameters on pressure loss and heat transfer coefficients based on the single factor changing, where the height, diameter, spacing and roughness of pin-fins are selected. Baby and Balaji ${ }^{[9]}$ conducted experiment to find out the optimal volume fraction of heat sink and the number of pin-fins separately for thermal performance. Several special designs were also proposed, Khalili Sadaghiani and $\operatorname{Koşar}^{[10]}$ investigated the effect of structure parameters by using dimple in pin-fin heat sink and optimized the geometry based pattern research method. Generally, most investigations of pin-fin performance emphasized the influence of the single geometry parameter. Parameters of pin-fin geometry. Meanwhile, limited researcher comprehensively considers the influence of multiple factors on heat transfer and pressure loss based on uniform design (UD) ${ }^{[11]}$.

In this study, based on response surface method numerical simulation is conducted to investigate 
the interaction of height and diameter of pin-fin on the thermal and hydraulic performance of cylindrical aluminum pin-fin array arranging inline. Aims to optimized the thermal and hydraulic performance under the combined action of height and diameter of pin-fins. Equivalent thermal resistance and pressure loss used value to evaluate the effect of geometry modification based on interaction of pin-fin height and diameter change.

\section{Modeling of Turbulent Flow}

In this study cylindrical pin-fin was arranged in line. Diameter and height of pin-fins presented by $\mathrm{D}$ and $\mathrm{H}$ respectively, were selected as variables for investigation. The height, width and length of water channel were set as constant. In order to simulate the condition of model, several assumptions are made for analysis: (1) fluid is steady, turbulent and incompressible in 3D; (2) the velocity at inlet is uniform; (3) thermal properties of materials and fluid is constant; (4) the influence of viscous dissipation is not considered; (5) the radiation heat transfer is negligible.

There are several flow models based on two equations method for hydraulic-thermal simulation, such as k- $\varepsilon, \mathrm{k}-\omega$, Reynolds stress model[12]. K- $\omega$ model is applied to the transition or flow with low Reynolds ratio; Reynolds stress model is applied for complicated flow especially swirling; k- $\varepsilon$ generally is an empirical formula widely used in flow with high Reynolds ratio. In this study the standard $\mathrm{k}-\varepsilon$ model is adopted to simulate the turbulence model flow characteristics, the governing equation according to viscous model with assumptions are ${ }^{[6]}$ :

Mass equation:

$$
\partial u i \partial x i=0
$$

Momentum equation:

$$
\rho u_{j} \frac{\partial u_{i}}{\partial x_{j}}=-\frac{\partial p}{\partial x_{i}}+\frac{\partial}{\partial x_{j}}\left[\mu_{t}\left(\frac{\partial u_{i}}{\partial x_{j}}+\frac{\partial u_{j}}{\partial x_{i}}\right)\right]
$$

Transport equations for the standard k- $\varepsilon$ model:

$$
\begin{array}{r}
\frac{\partial}{\partial x_{j}}\left(\rho k u_{j}\right)=\frac{\partial}{\partial x_{j}}\left[\left(\mu+\frac{\mu_{t}}{\sigma_{k}}\right) \frac{\partial k}{\partial x_{j}}\right]+\mu_{t}\left(\frac{\partial u_{i}}{\partial x_{j}}+\frac{\partial u_{j}}{\partial x_{i}}\right) \frac{\partial u_{i}}{\partial x_{j}}-\rho_{\varepsilon} \\
\frac{\partial}{\partial x_{j}}\left(\rho \varepsilon u_{j}\right)=\frac{\partial}{\partial x_{j}}\left[\left(\mu+\frac{\mu_{t}}{\sigma_{\varepsilon}}\right) \frac{\partial \varepsilon}{\partial x_{j}}\right]-\rho C_{2} \frac{\varepsilon^{2}}{k_{f}}+C_{1} \frac{\varepsilon}{k_{f}} \mu_{t}\left(\frac{\partial u_{i}}{\partial x_{j}}+\frac{\partial u_{j}}{\partial x_{i}}\right) \frac{\partial u_{i}}{\partial x_{j}}
\end{array}
$$

Where $u_{i}$ and $u_{j}$ are the velocity of the $x_{i}$ and $x_{j}$ direction based on average of time, respectively; $\rho$ is the density of the fluid, unit $(\mathrm{kg} / \mathrm{m} 3)$; $\mathrm{p}$ is average of pressure based on time, unit (Pa); $\mu$ and $\mu_{t}$ are dynamic viscosity for laminar flow and turbulent flow, respectively, unit $(\mathrm{kg} /(\mathrm{m} \cdot \mathrm{s}))$; $\mathrm{k}$ and $\varepsilon$ are turbulence kinetic energy and turbulent dissipation rate; C1 and C2 are constant value (1.44 and 1.92) $\sigma_{\varepsilon}$ and $\sigma_{k}$ are turbulent Prandtl number for $\varepsilon$ and $\mathrm{k}$ (1.3 and 1.0), respectively; $k_{f}$ is the thermal conductivity of fluid, unit $(\mathrm{W} /(\mathrm{m} \cdot \mathrm{K}))$.

In order to avoid the influence of backflow and region of turbulence developing are, front and back of pin-fin area is extended in the x-direction. The velocity and pressure at inlet and outlet are assumed to distribute uniformly. The thermal boundary conditions except for the interface of solid-liquid and heating surface, are perfectly adiabatic. The detailed physical model formula of boundaries is illustrated below:

The flow boundary conditions at the inlet are: $T_{i n}=T_{e}=300 \mathrm{~K}, u_{s}=0.3$

The thermal condition on the interface of solid-fluid surfaces where the temperature and heat flux $\left(1000 \mathrm{~W} / \mathrm{m}^{2}\right)$ on the top of heat sink are considered to be continuous and other boundaries are considered to be perfect insulated: $k_{s} \frac{\partial T_{s}}{\partial n}=k_{f} \frac{\partial T}{\partial n}, T_{s}=T$

Where $k_{s}$ and $k_{f}$ are thermal conductivity of solid and fluid, respectively; $T_{s}$ and $\mathrm{T}$ are solid temperature and fluid temperature at the contact surface, respectively; $\mathrm{n}$ is the normal direction of the contact surface. 
The flow condition at the outlet are: $P=P_{\text {out }}, \frac{\partial T}{\partial n}=0$

In this study, pressure loss and thermal resistance are selected to evaluate the hydraulic performance and heat transfer efficiency of the heat sink. The pressure loss $\left(P_{l}\right)$ and the thermal resistance $\left(R_{t}\right)$ of heat sink based on the average pressure and temperature difference between inlet and outlet of water passage, which indicates the hydraulic feature of heat sink, are defined by:

$$
\begin{array}{r}
P_{l}=\Delta P=\bar{P}_{\text {inlet }}-\bar{P}_{\text {outlet }} \\
R_{t}=\frac{\Delta T}{Q}=\frac{\bar{T}_{\text {out }}-\bar{T}_{\text {inlet }}}{Q}
\end{array}
$$

Where: $\bar{P}_{\text {inlet }}$ and $\bar{P}_{\text {outlet }}$ are the average pressure at inlet and outlet, respectively, unit (Pa); $\bar{T}_{\text {inlet }}$ and $\bar{T}_{\text {outlet }}$ are the average temperature at inlet and outlet, respectively, unit (K), Q is total heat flux on the top, unit $(\mathrm{W} / \mathrm{m} 2)$

\section{Optimization Design Based On RSM}

Optimization adopts response surface method (RSM) that bases on mathematics and statistics to investigate the influence of several variables on object responses. RSM is proposed by Box and Wilson originally to optimize the results of experiment. Generally, if the response is non-linear function of variables, a polynomial of high degree, such as second-order model, should be adopted instead of first-order model in the case of linear function. In this study, in order to optimize the responses, quadratic model which should be rotatable is established.

Central composite design (CCD) is a most widely used method of RSM to figure out the relationship between responses and variables. In CCD, each factor should have three levels for analysis and in order to meet the rotatability, $\alpha=\left(n_{F}\right)^{1 / 4}$ where the value of $\alpha$ depends on the number of points in the factorial portion of the design and $n_{\mathrm{F}}$ is the number of the points.

$$
\mathrm{y}=\beta_{0}+\sum_{i=1}^{k} \beta_{i} x_{i}+\sum_{i=1}^{k} \beta_{i i} x_{i}^{2}+\sum_{i} \sum_{j} \beta_{i j} x_{i} x_{j}+\varepsilon
$$

Where: $\mathrm{y}$ is target response, $x_{i}$ and $x_{j}$ are the coded values of defined variables; $\mathrm{i}$ is linear coefficient; $\mathrm{j}$ is quadratic coefficient; $\beta$ is regression coefficient; $\varepsilon$ is an accidental error.

According to the objectives of this study, the thermal resistance and the pressure loss are selected as the response. Height and diameter of pin-fin are set as variables, where the range of height is between 1-9 mm and the range of diameter increases from 0.5 to $3.5 \mathrm{~mm}$, and $\alpha=1.414$. Therefore, design point of simulation is list in Table 1:

Table 1 Independent variables with different level.

\begin{tabular}{|c|c|c|c|}
\hline Factors & Height $(\mathrm{H})$ & Diameter $(\mathrm{D})$ & standard variable $(\alpha)$ \\
\hline \multirow{4}{*}{ level $(\mathrm{mm})$} & 1 & 0.5 & -1.414 \\
\cline { 2 - 4 } & 2.172 & 0.039 & -1 \\
\cline { 2 - 4 } & 4 & 2 & 0 \\
\cline { 2 - 4 } & 7.828 & 3.06 & 1 \\
\cline { 2 - 4 } & 9 & 3.5 & 1.414 \\
\hline
\end{tabular}

\section{Numerical Example}

\subsection{Modeling}

The model is illustrated in Fig.1, the height and diameter of pin-fin in both solid plate and the fluid phase are variable, in addition, structure mesh around pin-fin was adopted to improve the accuracy of simulation. 

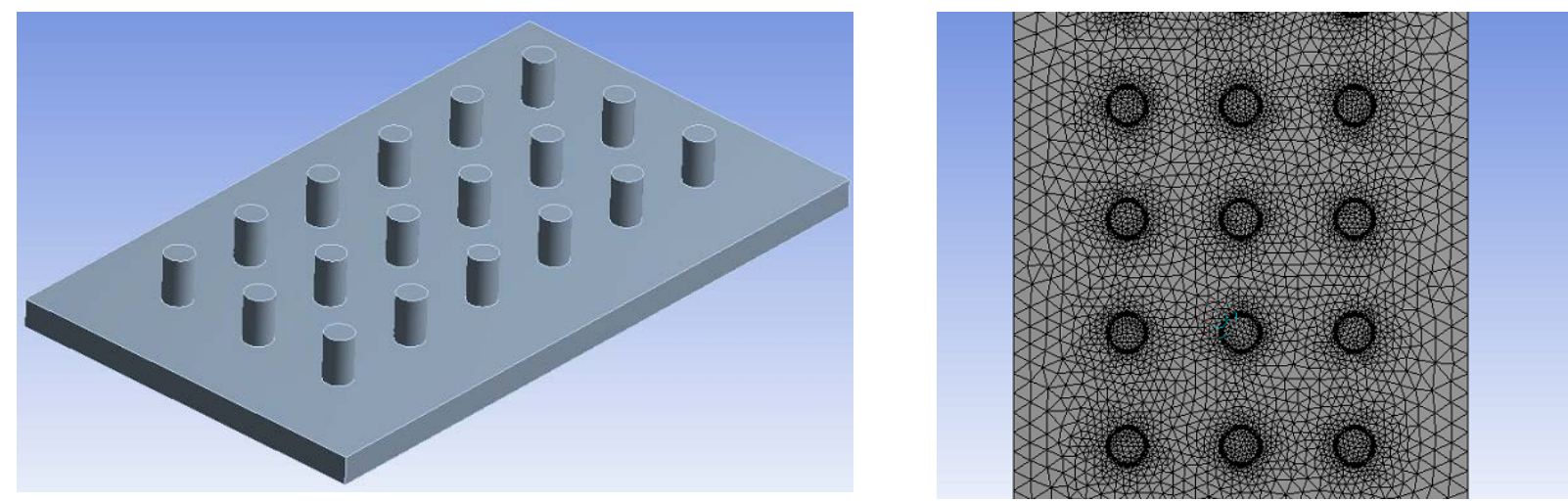

Fig.1 The 3D model of plate and mesh of fluid phase.

\subsection{Results and Discussion}

RSM is applied in this study not only to optimize the geometry of pin-fin also to investigate the interaction effects of designed factors on target response, namely pressure loss $\left(P_{l}\right)$ and thermal resistance $\left(R_{t}\right)$.

The response surface diagram in Fig. 2 shows the changing tendency of pressure loss with varying height and diameter of pin-fin, where the pressure loss increases continuously with increase of height and diameter of pin-fin. In addition, response surface also indicates the change of pressure loss is gradual when the change of height is unobvious, which indicates the influence of height on pressure loss is greater than that of the diameter. The minimum pressure loss is $0.181 \mathrm{~Pa}$ with $1 \mathrm{~mm}$ height and $1.85 \mathrm{~mm}$ diameter, maximum pressure loss is $0.328 \mathrm{~Pa}$ with $9 \mathrm{~mm}$ height and $3.5 \mathrm{~mm}$ diameter.
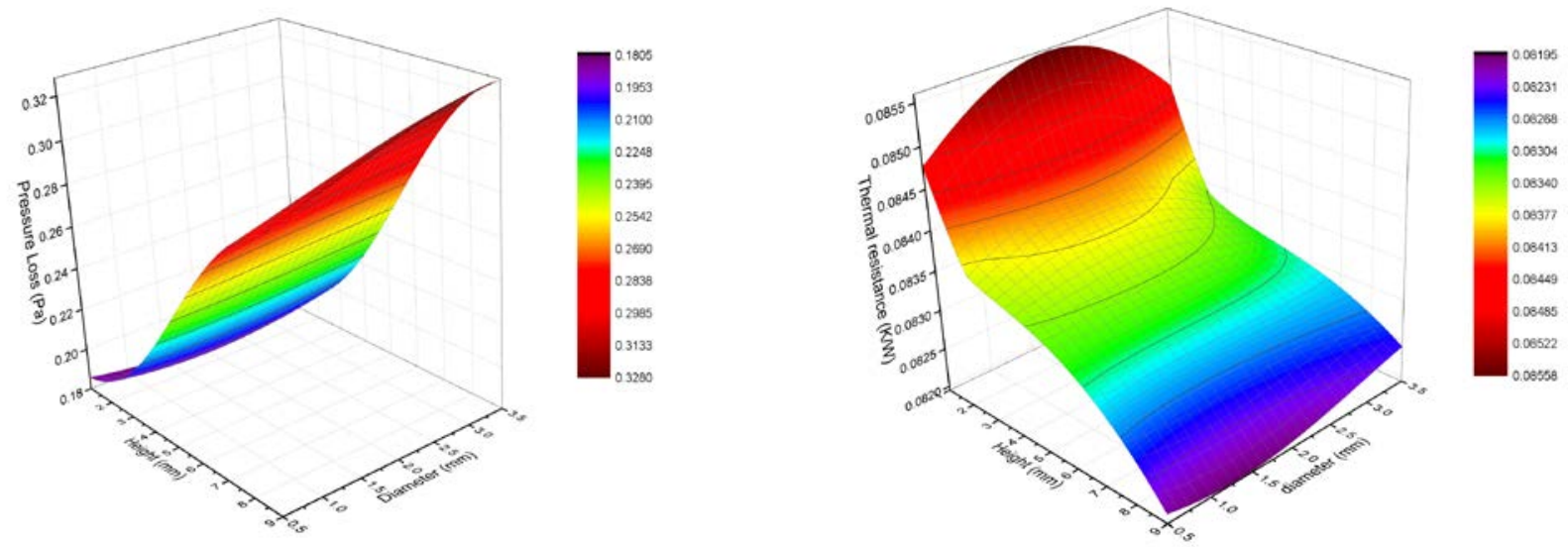

Fig.2 3D response surface plot of height and diameter of pin-fin for pressure loss; 3D response surface plot of height and diameter for thermal resistance.

Almost opposite results of response surface for thermal resistance are achieved in Fig.2. The plot shows the interaction effects of height and diameter on the thermal resistance. The effect of diameter on thermal resistance is similar to the response of pressure loss, when height varies slightly, the change of thermal resistance caused by varying of the diameter is not obvious. However, the changing of pressure loss is not remarkable when height of pin-fin is in the range of $3 \mathrm{~mm}$ to $7 \mathrm{~mm}$. Nevertheless, when the range of height exceeds this region, the change of thermal resistance is rapid. The minimum thermal resistance is $0.082 \mathrm{~K} \cdot \mathrm{m}^{2} / \mathrm{W}$ with $9 \mathrm{~mm}$ height and $1.3 \mathrm{~mm}$. The maximum thermal resistance is $0.086 \mathrm{~K} \cdot \mathrm{m}^{2} / \mathrm{W}$ with $1 \mathrm{~mm}$ height and $2 \mathrm{~mm}$ diameters.

In order to investigate the effect of each variables on response value and interaction of variables for response value, sensitivity analysis is used for this study, and sensitivity is defined below:

$$
\text { sensitivity }(\%)=\frac{\partial R_{t}}{\partial H}
$$

The sensitivity of each response with corresponding variables is $\frac{\partial R_{t}}{\partial H}, \frac{\partial P_{l}}{\partial H}, \frac{\partial R_{t}}{\partial D}$, and $\frac{\partial P_{l}}{\partial D}$. Meanwhile, the positive sensitivity means variables have a positive relationship with objectives, in 
opposite, the negative sensitivity means response value is negative relating to independent variables.

By comparing the results of Fig.3, the sensitivity of the thermal to both height and diameter is more than the sensitivity of pressure loss to these two parameters. Furthermore, the sensitivity of thermal resistance to the height is greater than thermal resistance to diameter; while the sensitivity of pressure loss to height is slightly less than the sensitivity thermal resistance to diameter. On the other hand, when increasing the height, the sensitivity of thermal resistance and pressure loss to diameter increase, particularly, the sensitivity increases from negative to positive value.

In summary, numerical simulation is conducted to achieve the optimal value of $R_{t}$, plan to list the results of temperature distribution and hydraulic condition, compare the initial design with optimized design, but the results in Fluent are not obvious. Comparing initial designed point with optimal point when thermal resistance is selected as target, the reduction of thermal resistance is around $4.7 \%$, pressure loss between optimal design and initial design decreases by $44.8 \%$.
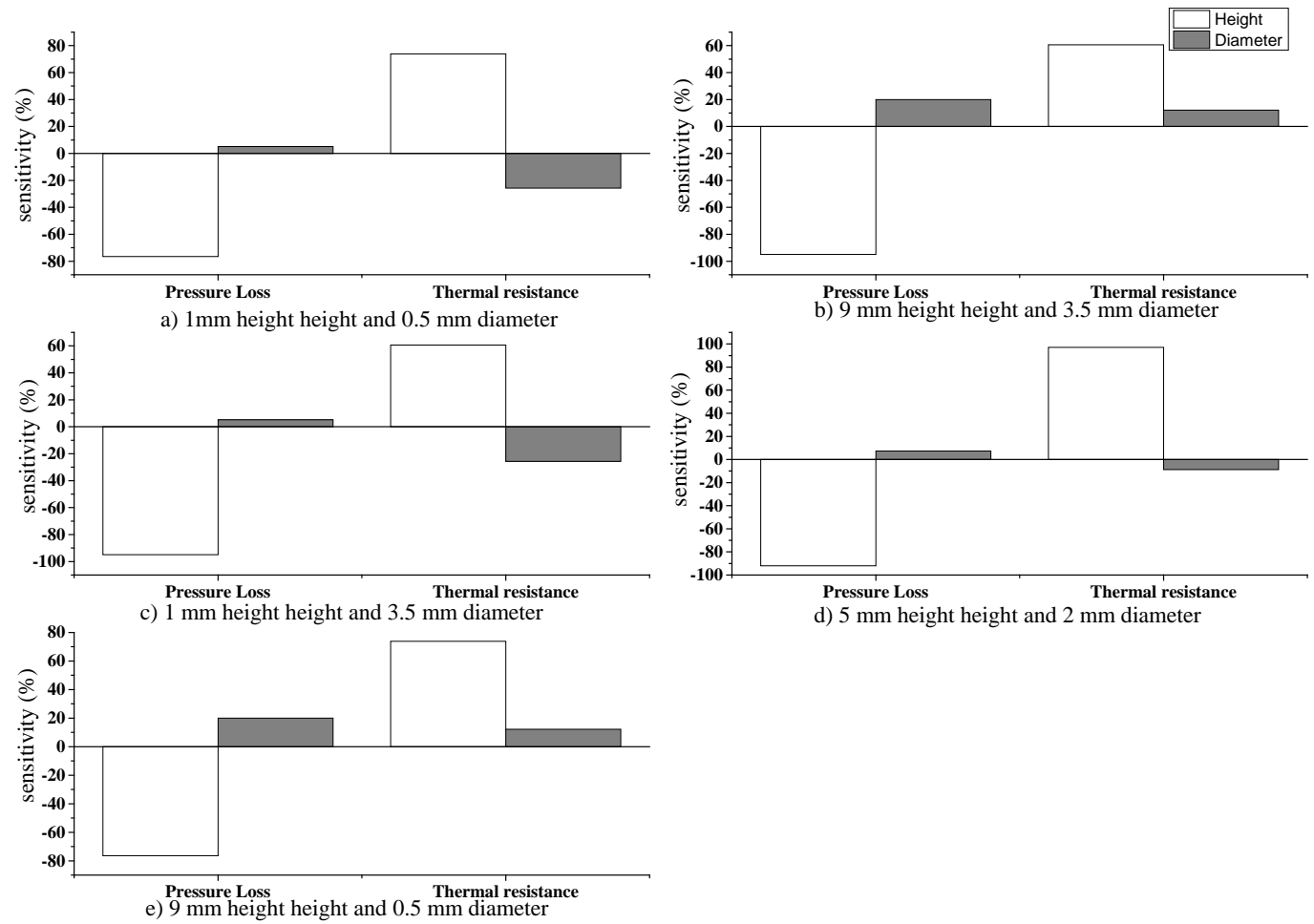

d) $5 \mathrm{~mm}$ height height and $2 \mathrm{~mm}$ diameter

Fig.3 Sensitivity analysis results of pressure loss and thermal resistance with corresponding variables (height, diameter): a) (1 mm, $0.5 \mathrm{~mm})$; b) (9 mm, $3.5 \mathrm{~mm})$; c) (1 mm, $3.5 \mathrm{~mm})$; d) (5 mm, $2 \mathrm{~mm})$; e) (9 mm, $0.5 \mathrm{~mm})$.

\section{Conclusion}

In order to improve the hydraulic and thermal performance of pin-fin in heat sink, the appropriate turbulent models were studied and standard $k-\varepsilon$ model was used for the coupled simulation of thermal and fluid analysis. The RSM was approved to be an efficient numerical approach in multi-factors optimization design. The simulation results indicated the pressure loss increased when the height and diameter increased, additionally the sensitivity of pressure loss to height was greater than that of pressure loss to diameter; withal there was obvious enhancing tendency for thermal resistance when height of diameter increase, however, the influence of diameter on thermal resistance was positive in a certain range while effect was opposite within other range.

\section{Acknowledgement}

This work was carried out with support from the Jiangsu Science and Technology Programme (Grant No. BK20170418) and the University Natural Science Research Programme of Jiangsu 
Province (Grant No. 16KJB460021).

\section{Reference}

[1] Garimella S V, Fleischer A S, Murthy J Y, et al. Thermal challenges in next-generation electronic systems[J]. IEEE Transactions on Components and Packaging Technologies, IEEE, 2008, 31(4): 801-815.

[2] Ricci R, Montelpare S. An experimental IR thermographic method for the evaluation of the heat transfer coefficient of liquid-cooled short pin fins arranged in line[J]. Experimental Thermal and Fluid Science, 2006, 30(4): 381-391.

[3] Shuja S Z. Optimal fin geometry based on exergoeconomic analysis for a pin-fin array with application to electronics cooling[J]. Exergy, An International Journal, 2002, 2(4): 248-258.

[4] Rubio-Jimenez C A, Kandlikar S G, Hernandez-Guerrero A. Performance of online and offset micro pin-fin heat sinks with variable fin density[J]. IEEE transactions on components, packaging and manufacturing technology, IEEE, 2013, 3(1): 86-93.

[5] Zhao J, Huang S, Gong L, et al. Numerical study and optimizing on micro square pin-fin heat sink for electronic cooling[J]. Applied Thermal Engineering, 2016, 93: 1347-1359.

[6] Yang A, Chen L, Xie Z, et al. Constructal heat transfer rate maximization for cylindrical pin-fin heat sinks[J]. Applied Thermal Engineering, 2016, 108: 427-435.

[7] Wei X, Joshi Y, Patterson M K. Experimental and Numerical Study of a Stacked Microchannel Heat Sink for Liquid Cooling of Microelectronic Devices[J]. Journal of Heat Transfer, ASME, 2007, 129(10): 1432-1444.

[8] Wan Z, Joshi Y. Pressure drop and heat transfer characteristics of square pin fin enhanced microgaps in single phase microfluidic cooling[A]. 14th InterSociety Conference on Thermal and Thermomechanical Phenomena in Electronic Systems, ITherm 2014[C]. G. W. Woodruff School of Mechanical Engineering, Georgia Institute of Technology, 801 Ferst Dr, Atlanta, GA, United States: Institute of Electrical and Electronics Engineers Inc., 2014: 649-657.

[9] Baby R, Balaji C. Thermal optimization of PCM based pin fin heat sinks: An experimental study[J]. Applied Thermal Engineering, 2013, 54(1): 65-77.

[10] Li P, Luo Y, Zhang D, et al. Flow and heat transfer characteristics and optimization study on the water-cooled microchannel heat sinks with dimple and pin-fin [J]. International Journal of Heat and Mass Transfer, 2018, 119: 152-162.

[11] Yang J, Li L, Yang L, et al. Uniform design for the parameters optimization of pin-fins channel heat sink [J]. Applied Thermal Engineering, 2017, 120(Supplement C): 289-297.

[12] Wilcox D C. Turbulence modeling for CFD [M]. DCW industries La Canada, CA, 1993, 2. 